\title{
REVIEW
}

Open Access

\section{Circulating chemerin levels in metabolic- associated fatty liver disease: a systematic review and meta-analysis}

Qian Ren ${ }^{1,2,3,4 \dagger}$, Hongya Wang ${ }^{1,2,3,4+}$, Yan Zeng ${ }^{1,2,3,4}$, Xia Fang ${ }^{1,2,3,4,5}$, Mei Wang ${ }^{1,2,3,4}$, Dongze Li $i^{1,2,3,4}$, Wei Huang ${ }^{1,2,3,4^{*}}$ and Yong $X u^{1,2,3,4^{*}}$

\begin{abstract}
Background and objectives: Chemerin is a brand-new adipokine that has been linked to both inflammation and metabolic dysfunction. Even though a rising number of studies have connected chemerin to metabolic-associated fatty liver disease (MAFLD), formerly referred to as non-alcoholic fatty liver disease (NAFLD), this association has been controversial.
\end{abstract}

Methods: A comprehensive literature search was undertaken up to February 1, 2022, in the PubMed, Embase, Web of Science, CNKI, WANFANG, and CBM library databases. Circulating chemerin levels were obtained and summarized using the standardized mean difference (SMD) and 95\% confidence interval (CI). Subgroup and meta-regression analyses were conducted to examine the possibility of heterogeneity.

Results: A total of 17 studies involving 2580 participants (1584 MAFLD patients and 996 controls) evaluated circulating chemerin levels in patients with MAFLD. The present study showed that higher chemerin levels were found in patients with MAFLD (SMD: 1.32; 95\% Cl: 0.29, 2.35) and nonalcoholic fatty liver (NAFL) (SMD: 0.75; 95\% Cl: $0.01,1.50)$ compared to controls. However, circulating chemerin levels did not differ significantly in the following comparisons: nonalcoholic steatohepatitis (NASH) patients and controls (SMD: 0.75; 95\% Cl: -0.52, 2.03); NASH patients and NAFL patients (SMD: 0.16; 95\% Cl: $-0.39,0.70$ ); moderate to severe steatosis and mild steatosis (SMD: 0.55; 95\% Cl: -0.59, 1.69); present liver fibrosis and absent liver fibrosis (SMD: 0.66; 95\% Cl: -0.42, 1.74); present lobular inflammation and absent lobular inflammation (SMD: 0.45; 95\% Cl: -0.53, 1.42); and present portal inflammation and absent portal inflammation (SMD: 1.92; 95\% Cl: -0.85, 4.69).

Conclusions: Chemerin levels were considerably greater in patients with MAFLD than in controls, despite the fact that they were not significantly linked to different liver tissue lesions of MAFLD. In different subtypes of MAFLD, in comparison to healthy controls, the chemerin levels of NAFL patients were higher, whereas, there was no obvious difference in chemerin levels between NASH patients and controls. It is possible that chemerin will be used as a biomarker in the future to track the development and progression of MAFLD.

Keywords: Chemerin, Adipokines, Nonalcoholic fatty liver disease, Metabolic- associated fatty liver disease, Metaanalysis

\footnotetext{
*Correspondence: huangwei1212520@163.com; xywyll@swmu.edu.cn

${ }^{\dagger}$ Qian Ren and Hongya Wang contributed equally to this work.

'Department of Endocrinology and Metabolism, The Affiliated Hospital of

Southwest Medical University, Luzhou, Sichuan, China

Full list of author information is available at the end of the article
}

(c) The Author(s). 2022 Open Access This article is licensed under a Creative Commons Attribution 4.0 International License, which permits use, sharing, adaptation, distribution and reproduction in any medium or format, as long as you give appropriate credit to the original author(s) and the source, provide a link to the Creative Commons licence, and indicate if changes were made. The images or other third party material in this article are included in the article's Creative Commons licence, unless indicated otherwise in a credit line to the material. If material is not included in the article's Creative Commons licence and your intended use is not permitted by statutory regulation or exceeds the permitted use, you will need to obtain permission directly from the copyright holder. To view a copy of this licence, visit http://creativecommons.org/licenses/by/4.0/. The Creative Commons Public Domain Dedication waiver (http://creativecommons.org/publicdomain/zero/1.0/) applies to the data made available in this article, unless otherwise stated in a credit line to the data. 


\section{Introduction}

Nonalcoholic fatty liver disease (NAFLD), one of the most common causes of end-stage liver disease, was redefined as metabolic-associated fatty liver disease (MAFLD) in recent years [1], and 25\% of the world's population is believed to be impacted by this disease according to current estimates [2,3]. By 2030, it is anticipated that the prevalence of MAFLD will increase from 17.6 to $22.2 \%$ in China and from 26.3 to $28.4 \%$ in the United States, while in other European countries, such as Italy, the prevalence is nearly $30 \%$ [4]. MAFLD is primarily defined by lipid accumulation while excluding some other causes of lipid accumulation, such as severe alcohol intake, drug use, and genetic illnesses. MAFLD includes a series of disease processes with fatty deposits in the liver, varying from simple steatosis [nonalcoholic fatty liver (NAFL)] to nonalcoholic steatohepatitis (NASH), which gradually causes liver fibrosis and may eventually progress to cirrhosis/liver failure or even liver cancer $[5,6]$. In addition to the effects on the liver itself, there is a lot of evidence suggesting that MAFLD may be related to metabolic diseases [obesity, type 2 diabetes mellitus (T2DM), hypertension, hyperlipidemia, metabolic syndrome] and extrahepatic tumors [7-9]. These adverse effects place a significant economic/health burden on society as a whole and humanity [10]. It is crucial to identify and diagnose MAFLD patients. Nevertheless, until now, the gold standard for diagnosing MAFLD has been liver biopsy, which has drawbacks including invasiveness, poor acceptability, sampling variability, and even the possibility of life-threatening complications [11]. Therefore, noninvasive biomarkers are essential for the identification of MAFLD.

Chemerin, also called retinoic acid receptor responder 2 (RARRES2) or tazarotene inducible gene 2 (TIG2), is a novel $14 \mathrm{kDa}$ chemotactic protein that was initially found in psoriasis and later defined as a novel adipokine that is widely present in the liver and adipose tissue and closely related to multiple physiological activities, such as innate immunity, inflammation, endothelial dysfunction, metabolic disorders, and angiogenesis [12-15]. Experiments have shown that exogenous chemerin can exacerbate glucose tolerance and reduce serum insulin levels [16-18]. Emerging evidence has suggested that has been linked to a number of pro-inflammatory cytokines (such as TNF- $\alpha, \mathrm{C}$-reactive protein, and $I L-6)[19,20]$. More recently, it has been established that serum chemerin levels can be used as an indicator of the efficacy of anti-inflammatory therapy for inflammatory bowel disease [21]. Chronic inflammation and insulin resistance have been identified as the most pathogenesis of MAFLD [22, 23]. Given the role of chemerin in regulating insulin levels and chronic inflammation, we boldly speculate whether its level is related to MAFLD. Several studies have shown a correlation between MAFLD and chemerin [24-34], but an association has not been found in other studies between chemerin levels and MAFLD [35-38]. Moreover, changes in serum chemerin have been reported in MAFLD patients with different subtypes by previous studies [24, 33, 36, 37, 39]. Similarly, the effects of circulating chemerin levels on hepatic steatosis, fibrosis, and inflammation have been investigated in several studies [24, 36, 39]. It's still not clear what the role of circulating chemerin is in MAFLD, though. Here, a comprehensive review and meta-analysis was conducted to explore the link between chemerin levels and MAFLD (NAFL or/and NASH) and its different liver tissue lesions, including hepatic steatosis, liver fibrosis, lobular inflammation, and portal inflammation, which may provide clues for the diagnosis and treatment of MAFLD.

\section{Methods \\ Methods and literature search}

This meta-analysis was performed following a predefined plan and recorded in accordance with PRISMA guidelines [40] (see Supplementary Additional File 2), and it has been added to the PROSPERO database, which is the International Register of Systemic Reviews (No. CRD42021290744).

Researchers (QR and HYW) combed PubMed, EMBASE, Web of Science, CNKI, WANFANG, and CBM library databases from their inception up to February 1, 2022. The search terms were based on subject terms and free terms related to chemerin and MAFLD, such as ('fatty liver' OR 'liver, nonalcoholic fatty' OR 'steatohepatitis, nonalcoholic' OR 'steatohepatitis, nonalcoholic' OR 'NASH' OR 'nonalcoholic fatty liver disease' OR 'NAFLD' OR 'MAFLD' OR 'metabolic-associated fatty liver disease' OR 'nonalcoholic fatty liver disease' OR 'nonalcoholic fatty liver' OR 'nonalcoholic steatohepatitis' OR 'steatosis') And ('chemerin'). The specific search strategy is shown in Supplemental Table 1. In addition, a manual search using a reference list of relevant articles was added. The search was not restricted by region or language. The reviewers resolved any disagreement through discussion.

\section{Inclusion and exclusion criteria}

A protocol to answer two $\mathrm{PI}(\mathrm{E}) \mathrm{CO}$ questions was performed:

(1) "Are circulating chemerin levels increased in patients with MAFLD (NAFL and/or NASH) compared to healthy individuals?"

(2) "Are there differences in chemerin levels between groups of specific histological lesions in patients with MAFLD?"

The questions had the following statements: 
(1) Otherwise healthy patients (Patients, P); MAFLD (NAFL or/and NASH) (Exposure, E); Non-MAFLD (Comparison, C); circulating chemerin levels (Outcome, $\mathrm{O})$.

(2) MAFLD patients (Patients, P); A specific histopathological lesion of the liver (Exposure, E); No corresponding specific histopathological lesion of the liver (Comparison, C); circulating chemerin levels (Outcome, $\mathrm{O})$.

Cohort studies, cross-sectional studies, and casecontrol studies were all considered for inclusion if they met the following standards: (1) participants in the case group were diagnosed with MAFLD (NAFL or/and $\mathrm{NASH}$ ) by various means, including ultrasound and biopsy; (2) participants in the control group were healthy; (3) circulating levels of chemerin were reported; (4) subjects aged $>18$ years were involved; or (5) investigation of the relationship of circulating chemerin with different liver histological lesions such as liver steatosis, liver fibrosis, lobular inflammation and portal inflammation.

Articles were excluded if they (1) studied chronic liver disease or cirrhosis caused by other factors, such as alcohol use, viruses, or autoimmune factors; (2) studied other liver diseases that coexist with MAFLD, such as hepatocellular tumors; or (3) were reviews, editorials, letters, case reports, comments, or conference abstracts.

\section{Quality assessment and data extraction}

The Risk of Bias of all included studies was assessed by QR and HYW independently. The risk of bias in the literature included in this study was assessed using the Risk of Bias in Non-Randomized Exposure Studies (ROBINS-E) tool [41]. In addition, the grading of recommendations, assessment, development and evaluation (GRADE) methodology was utilized to assess the quality of our studies [42].

The extracted data included information on trial characteristics (first author, publication year, country, sample size, study design, method of chemerin measurement, sample type, and diagnosis method of MAFLD), baseline information of the participants [age, sex, body mass index (BMI), aspartate aminotransferase (AST) levels, alanine aminotransferase (ALT) levels, and homoeostasis model assessment of insulin resistance (HOMA-IR)], and chemerin levels in patients with MAFLD (NAFL or/ NASH) and MAFLD specific liver histological lesions (mild, moderate and severe steatosis, presence and absence of liver fibrosis, lobular inflammation, and portal inflammation). If necessary, the corresponding author was contacted by email for more information to ensure that all relevant information was included. If the corresponding author did not respond, according to Cochrane's books and Hozo et al. [43], the means and standard deviations were obtained by converting the median and quartile forms of the chemerin levels.

\section{Analysis and statistics}

All statistical analyses were carried out with Stata 15.0 (StataCorp LP, College Station, TX, USA). The effect size was determined by the sample size, mean chemerin levels, and standard deviation (SD), and was presented as standardized mean difference (SMD) and 95\% confidence interval $(\mathrm{CI})$ of the circulating chemerin levels in comparisons between groups. Heterogeneity between studies was assessed using Cochrane $Q$ statistics, and heterogeneity levels were measured using $I^{2}$ statistics. Depending on the $I^{2}$ values, a score of $25 \%$ indicated moderate heterogeneity, whereas values of $50 \%$ and $75 \%$ indicated medium and high heterogeneity, respectively. A random-effects model was used when $I^{2}>50 \%$, conversely, a continuously weighted fixed-effects model was used. When the heterogeneity was high, subgroup and meta-regression analysis were used to evaluate the potential regulatory effect of the continuous variables on interstudy heterogeneity. To verify the firmness of the results, a sensitivity analysis omitting a single study from the primary analysis was conducted. Publication bias was evaluated by examining the symmetry of the funnel plot, and the significance of the symmetry was examined using Egger's linear regression test. A $P$ value $<0.05$ was deemed statistically significant unless otherwise indicated.

\section{Results}

Literature search

An initial search of 495 studies yielded a final total of 17 eligible reports that included 2580 participants (1584 MAFLD patients and 996 controls) that met the inclusion criteria. The results of our literature search are summarized in Fig. 1.

\section{Features of the included studies}

Table 1 and Supplementary Table 2 contain detailed characteristics and biochemical parameters of all the study subjects. Nine studies had cross-sectional designs $[26,27,29,35-39,44]$, and eight studies had casecontrol designs [24, 25, 28, 30-34]. Of all the articles, sixteen reported serum-derived chemerin levels [24-39], and only one reported plasma-derived chemerin levels [44]. These clinical tests were carried out in a variety of locations throughout the world: seven studies were conducted in Europe (Germany [36], Belgium [37], Greece [38], and France [44] conducted one each; three were conducted in Poland [24, 27, 39]); eight studies were conducted in Asia (one was conducted in Turkey [25], and seven were conducted in China [26, 28, 29, 31, 33- 


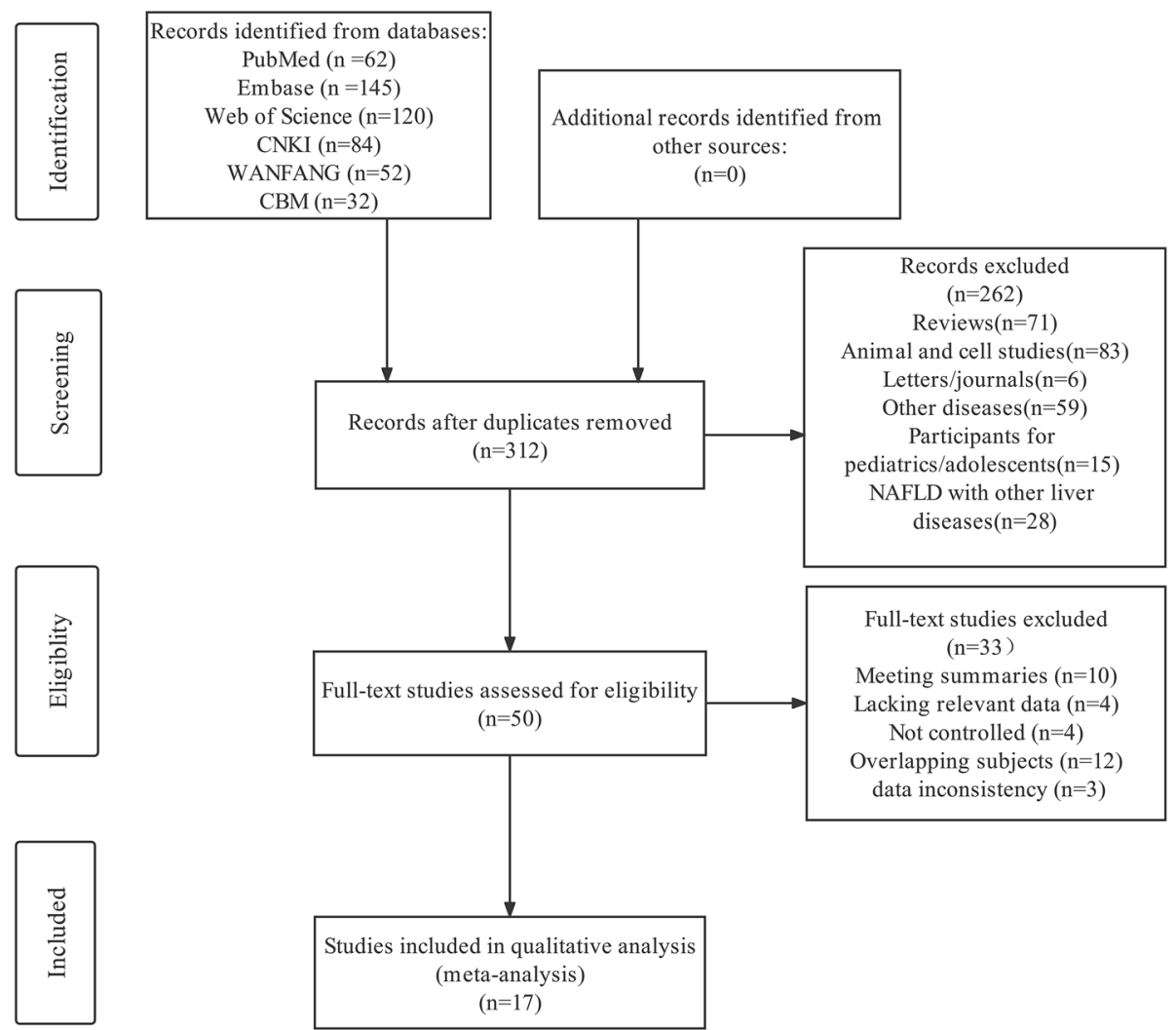

Fig. 1 PRISMA flow chart of the literature search process

Table 1 Details of the selected studies and baseline characteristics of the participants

\begin{tabular}{|c|c|c|c|c|c|c|c|}
\hline Authors & Year & Country & Sample size & Study design & Method of chemerin measurement & Sample & Diagnostic methods of MAFLD \\
\hline Kukla et al. & 2010 & Poland & 61 & Case-control & Enzyme-linked immunosorbent assay & Serum & Liver biopsy \\
\hline Sell et al. & 2010 & France & 44 & Cross-sectional & Enzyme-linked immunosorbent assay & Plasma & Liver biopsy \\
\hline Yilmaz et al. & 2011 & Turkey & 174 & Case-control & Enzyme-linked immunosorbent assay & Serum & Liver biopsy \\
\hline Polyzos et al. & 2014 & Greece & 59 & Cross-sectional & Enzyme-linked immunosorbent assay & Serum & Liver biopsy \\
\hline Ye et al. & 2014 & China & 903 & Cross-sectional & Enzyme-linked immunosorbent assay & Serum & Ultrasound \\
\hline Zhuang et al. & 2015 & China & 45 & Cross-sectional & Enzyme-linked immunosorbent assay & Serum & Ultrasound \\
\hline Zwolak et al. & 2016 & Poland & 45 & Cross-sectional & Enzyme-linked immunosorbent assay & Serum & Liver biopsy \\
\hline Bekaert et al. & 2016 & Belgium & 90 & Cross-sectional & Enzyme-linked immunosorbent assay & Serum & Liver biopsy \\
\hline Pohl et al. & 2016 & Germany & 56 & Cross-sectional & Enzyme-linked immunosorbent assay & Serum & Ultrasound \\
\hline Lai et al. & 2017 & China & 89 & Case-control & Enzyme-linked immunosorbent assay & Serum & Liver biopsy or Ultrasound \\
\hline Kajor et al. & 2017 & Poland & 56 & Cross-sectional & Enzyme-linked immunosorbent assay & Serum & Liver biopsy \\
\hline Zhang et al. & 2018 & China & 300 & Cross-sectional & Enzyme-linked immunosorbent assay & Serum & Liver biopsy \\
\hline Hang et al. & 2019 & China & 310 & Case-control & Enzyme-linked immunosorbent assay & Serum & Liver biopsy or Ultrasound \\
\hline Sahar et al. & 2019 & Egypt & 45 & Case-control & Enzyme-linked immunosorbent assay & Serum & Ultrasound \\
\hline Mohamed et al. & 2021 & Egypt & 90 & Case-control & Enzyme-linked immunosorbent assay & Serum & Ultrasound \\
\hline Gao et al. & 2021 & China & 131 & Case-control & Enzyme-linked immunosorbent assay & Serum & Liver biopsy or Ultrasound \\
\hline Xing et al. & 2021 & China & 82 & Case-control & Enzyme-linked immunosorbent assay & Serum & Ultrasound \\
\hline
\end{tabular}


$35])$. Only two studies were conducted in Africa (Egypt [30, 32]). Eight studies used liver biopsy [24, 25, 27, 29, $36,37,39,44]$ to identify MAFLD, six studies used ultrasound technology [26, 30, 32, 34, 35, 38], and three studies were unclear about the method of determining a specific diagnosis of NAFL [28, 31, 33]. For the circulating chemerin levels in different types and pathological features of MAFLD, fifteen studies [24-29, 31-38] focused on MAFLD patients (1484 participants) and controls (996 participants); four studies [24, 30, 36, 37] focused on NAFL patients (107 participants) and controls (85 participants); four studies [24, 30, 36, 37] focused on NASH patients (76 participants) and controls (76 participants); five studies [24, 30, 36, 37, 39] concentrated on patients with NASH (93 participants) and NAFL (146 participants); three studies [24, 36, 44] investigated patients with mild and moderate steatosis and patients with severe steatosis (71 and 69 participants, respectively); four studies [24, 36, 39, 44] assessed patients with and without liver fibrosis (101 and 71 participants, respectively); three studies [36, 39, 44] assessed patients with and without lobular inflammation (63 and 68 participants, respectively); and three studies [24, 36, 44] assessed patients with and without portal inflammation (69 and 47 participants, respectively).

\section{Outcomes}

The random-effects model was adopted if a study had an $I^{2}>50 \%$. The results showed that patients with MAFLD had higher chemerin levels than the control group. (SMD: 1.32; 95\% CI: 0.29, 2.35, $P=0.012$, Fig. 2A), and chemerin levels were increased in NAFL patients relative to controls (SMD: 0.75 ; 95\% CI: 0.01, 1.50; $P=0.047$; Fig. 2B). However, significant heterogeneity was found in these studies $[\mathrm{Q}=1332.18$, degree of freedom $(\mathrm{df})=14$, $I^{2}=98.9 \%, P<0.001 ; \mathrm{Q}=13.58$, degree of freedom $(\mathrm{df})=$ $\left.3, I^{2}=77.9 \%, P=0.004\right]$. Conversely, there were no major differences in chemerin levels in NASH patients compared to controls. (SMD: 0.75; 95\% CI: $-0.52,2.03 ; P=$ 0.247; Fig. 2C) and NASH patients and NAFL patients (SMD: 0.16; 95\% CI: $-0.39,0.70 ; P=0.572$; Fig. 2D). Nevertheless, highly significant heterogeneity was still observed among these studies $[\mathrm{Q}=35.56$, degree of freedom $(\mathrm{df})=3, I^{2}=91.6 \%, P<0.001 ; \mathrm{Q}=15.57$, degree of freedom $\left.(\mathrm{df})=4, I^{2}=74.3 \%, P=0.004\right]$.

Equally interesting, there was no evident association between specific liver tissue lesions and chemerin levels in patients with MAFLD: moderate to severe steatosis and mild steatosis (SMD: 0.55; 95\% CI: $-0.59,1.69 ; P=$ 0.344; Fig. 3A), present liver fibrosis and absent liver fibrosis (SMD: 0.66; 95\% CI: -0.42, 1.74; $P=0.233$; Fig. 3B), present lobular inflammation and absent lobular inflammation (SMD: 0.45; 95\% CI: $-0.53,1.42 ; P=0.368$; Fig. 3C), and present portal inflammation and absent portal inflammation (SMD: 1.92; 95\% CI: $-0.85,4.69 ; P=$ 0.175; Fig. 3D). Again, we observed significant heterogeneity in these studies $[\mathrm{Q}=17.82$, degree of freedom $(\mathrm{df})=2, I^{2}=88.8 \%, P<0.001 ; \mathrm{Q}=29.27$, degree of freedom $(\mathrm{df})=3, I^{2}=89.7 \%, P<0.001 ; \mathrm{Q}=14.06$, degree of freedom $(\mathrm{df})=2, I^{2}=85.8 \%, P=0.001$; and $\mathrm{Q}=55.40$, degree of freedom $\left.(\mathrm{df})=2, I^{2}=96.4 \%, P<0.001\right]$.

Obvious heterogeneity was found in the present study. Owing to the limited number of studies, subgroup and meta-regression analysis were performed only when patients with MAFLD were compared with controls to identify possible sources of heterogeneity. Next, based on the mean age, study design, and region, subgroup analysis was conducted. MAFLD patients over the age of 50 had significantly lower circulating chemerin levels than controls (SMD: $-1.10 ; 95 \%$ CI: $-1.93,-0.27 ; P<$ 0.001 ; Table 2); conversely, patients under the age of 50 had elevated chemerin levels when compared to controls (SMD: 1.91; 95\% CI: 1.44, 2.39; $P=0.009$; Table 2). Significant heterogeneity was found in the age subgroup analysis $\left[\mathrm{Q}=25.14\right.$, degree of freedom $(\mathrm{df})=2, I^{2}=92 \%$, $P<0.001 ; \mathrm{Q}=126.73$, degree of freedom $(\mathrm{df})=11, I^{2}=$ 91.3\%, $P<0.001]$. In the subgroup analysis of the study design, higher levels of circulating chemerin were found in MAFLD patients than in controls in the case-control studies (SMD: 1.97; 95\% CI: $0.40, \quad 3.53 ; \quad P<0.001$; Table 2), whereas no significant differences were found in the cross-sectional studies (SMD: 0.48; 95\% CI: -1.01, 1.97; $P=0.526$; Table 2). A high degree of heterogeneity was observed in the case-control studies $[Q=109$, degree of freedom $\left.(\mathrm{df})=7, I^{2}=93.6 \%, P<0.001\right]$ and in cross-sectional studies $[Q=574.48$, degree of freedom $\left.(\mathrm{df})=6, I^{2}=99 \%, P<0.001\right]$. There was a significant trend of high chemerin protein levels in Asian subgroups of MAFLD patients (SMD: 1.73; 95\% CI: $0.35,3.10 ; P=$ 0.014; Table 2), whereas chemerin levels in the others did not differ from the control group in the regional subgroup analysis (SMD: 0.50; 95\% CI: $-0.61,1.60 ; P=$ 0.378 ; Table 2). A high degree of heterogeneity was still found in the regional subgroup analysis $[Q=1266.77$, degree of freedom $(\mathrm{df})=9, I^{2}=99.3 \%, P<0.001 ; \mathrm{Q}=$ 65.13, degree of freedom $\left.(\mathrm{df})=4, I^{2}=93.9 \%, P<0.001\right]$.

Unfortunately, no exact source of heterogeneity was found in the subgroup analysis between MAFLD patients and controls. Next, meta-regression according to sample size, sex ratio, mean age, mean BMI, mean AST levels, mean ALT levels, and mean HOMA-IR levels in patients with MAFLD was conducted. The results suggested that the heterogeneity in the data was caused in part by the mean age of the patients with MAFLD $\left(R^{2}=\right.$ 


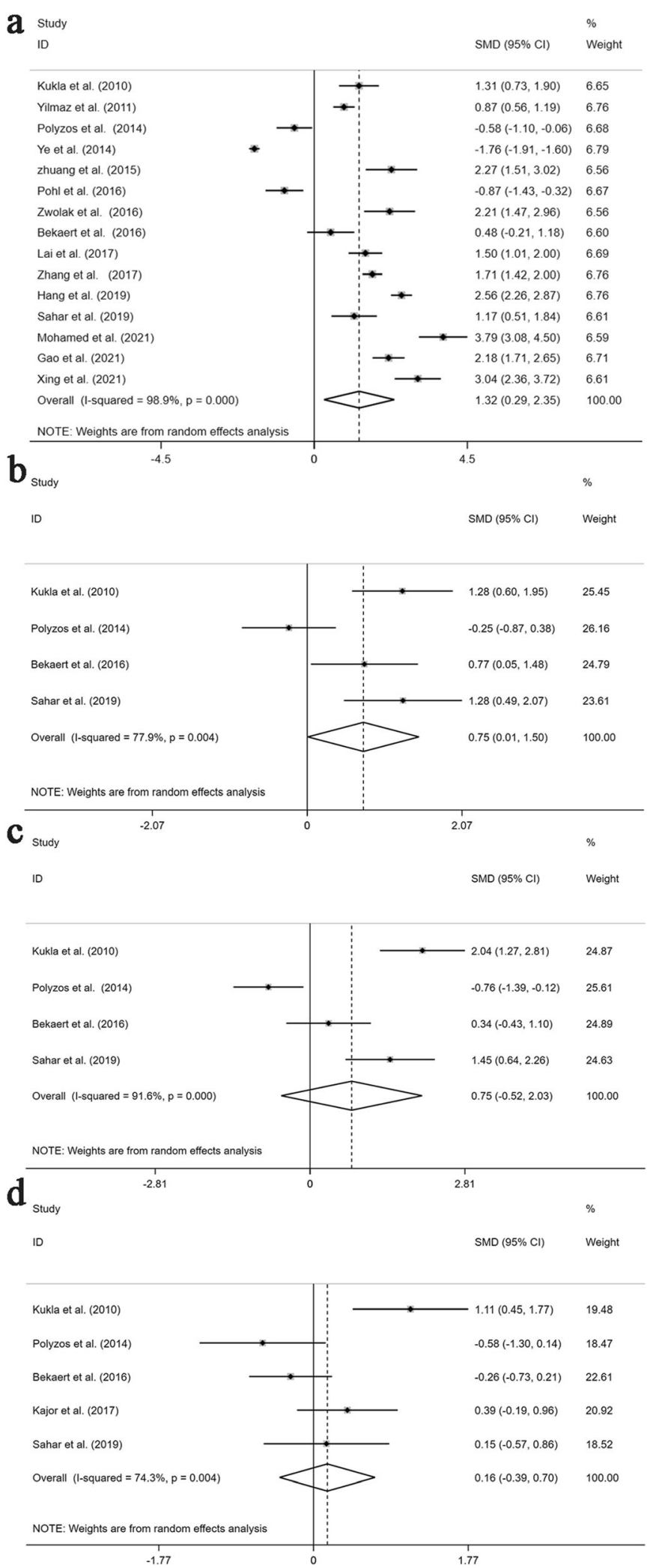

Fig. 2 Forest plots presenting the quantitative synthesis of circulating chemerin levels, comparing the following groups in the sum of the included studies: MAFLD patients and controls (A); NAFL patients and controls (B); NASH patients and controls (C); and NASH patients and NAFL patients (Meta-analysis) 


\section{a Study}

ID

SMD $(95 \% \mathrm{Cl}) \quad$ Weight

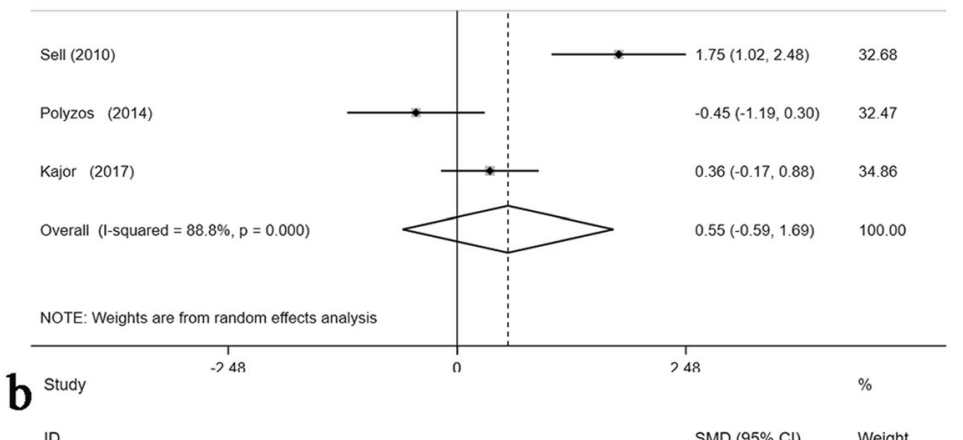

10

$\operatorname{SMD}(95 \% \mathrm{Cl}) \quad$ Weight

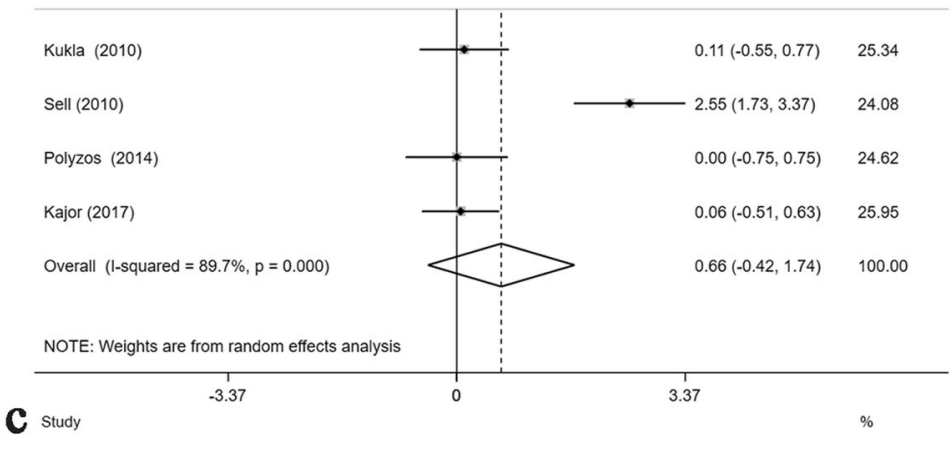

10

SMD $(95 \% \mathrm{Cl}) \quad$ Weight

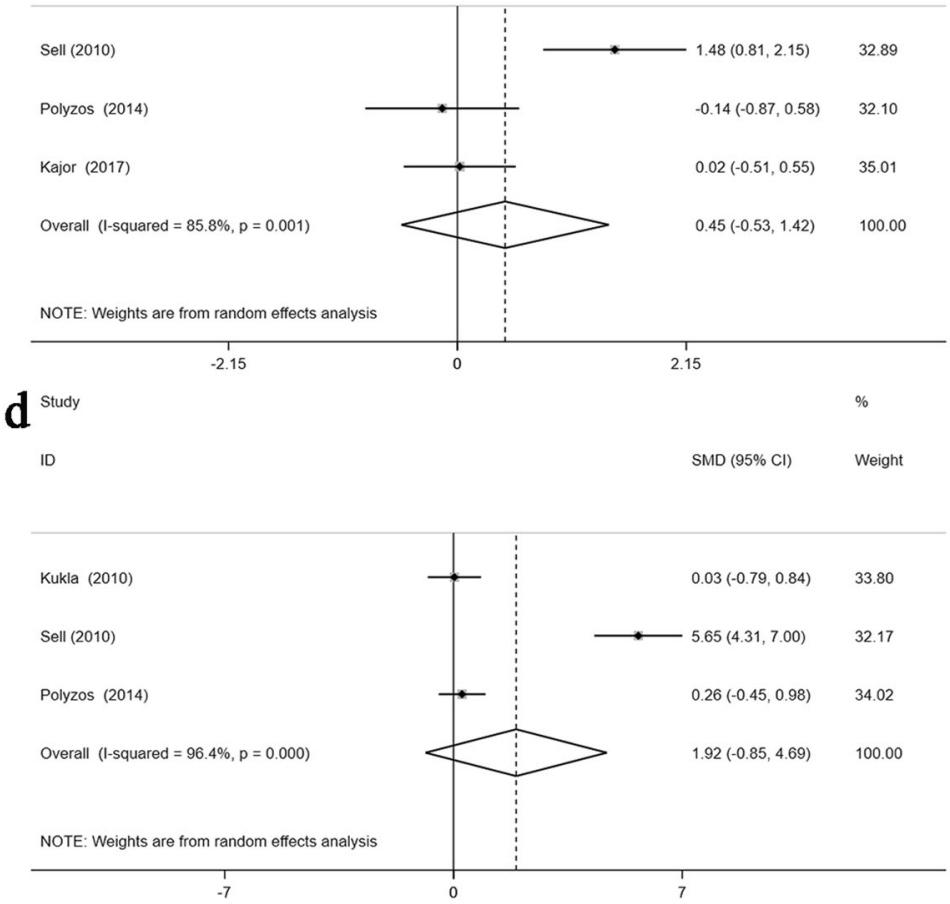

Fig. 3 Forest plots presenting the quantitative synthesis of circulating chemerin levels, comparing the following groups in the sum of the included studies: moderate to severe steatosis and mild steatosis (A); present liver fibrosis and absent liver fibrosis (B); present lobular inflammation and absent lobular inflammation (C); and present portal inflammation and absent portal inflammation (D) 
Table 2 Subgroup analysis of circulating chemerin levels in MAFLD patients compared with controls

\begin{tabular}{|c|c|c|c|c|}
\hline & \multicolumn{4}{|c|}{ All studies } \\
\hline & Studies & SMD $(95 \% \mathrm{Cl})$ & $P^{2}(\%)$ & $P$ for heterogeneity \\
\hline Overall & 15 & $1.32(0.29,2.35)$ & 98.9 & $<0.0001$ \\
\hline \multicolumn{5}{|c|}{ Subgroup analysis } \\
\hline \multicolumn{5}{|l|}{ Region } \\
\hline Asian & 10 & $1.73(0.35,3.10)$ & 99.3 & $<0.0001$ \\
\hline Others & 5 & $0.50(-0.61,1.60)$ & 93.9 & $<0.0001$ \\
\hline \multicolumn{5}{|l|}{ Mean age (y) } \\
\hline$<50$ & 12 & $1.91(1.44,2.39)$ & 91.3 & $<0.0001$ \\
\hline$\geq 50$ & 3 & $-1.10(-1.93,-0.27)$ & 92 & $<0.0001$ \\
\hline \multicolumn{5}{|l|}{ Study design } \\
\hline Case-control & 8 & $2.04(1.37,2.70)$ & 95.5 & $<0.0001$ \\
\hline Cross-sectional & 7 & $0.48(-1.01,1.97)$ & 99 & $<0.0001$ \\
\hline
\end{tabular}

Cl confidence interval, MAFLD metabolic-associated fatty liver disease, $S M D$ standardized mean difference

72.08\%; $\beta=-0.235 ; P<0.001$; Fig. 4 ), and other variables did not work in the heterogeneity exploration (Supplementary Table 3).

\section{Study quality assessment}

The risk of bias was assessed comprehensively for each of the included studies using the ROBINS-E tool (Supplementary Table 4). All qualified studies were judged to be low risk by rating the intended exposure, selection, missing data, measurement of outcomes, and reported results. Regarding confounding bias, nine studies were considered to be at serious risk [26, 27, 29, 35-39, 44], and eight studies [24, 25, 28, 30-34] were regarded as being at moderate risk. Considering exposure measurement bias, three studies were categorized as having a moderate risk of bias $[28,31,33]$, and fourteen studies [24-27, 29, 30, 32, $34-39,44]$ were at low risk. Overall, of all the studies, nine studies $[26,27,29,35-39,44]$ were judged to be at serious risk, and eight studies were categorized as being at moderate risk [24, 25, 28, 30-34].

The GRADE system assessed the certainty of this study's findings at a very low level (Supplementary Table 5). The certainty of the evidence was determined to be low due to the observational nature of the study. Among these studies, serious risk of bias as assessed by the ROBINS-E tool, serious inconsistency owing to significant heterogeneity between studies, imprecision due to the limited number of studies, and publication bias all contributed to the progressively lower certainty of evidence.

\section{Sensitivity and publication bias analyses}

Due to volume limitations, sensitivity and publication bias analyses were used only for studies comparing patients with MAFLD and controls. The sensitivity analyses showed that the results of a single study had little impact on the results of this meta-analysis study (Fig. 5).

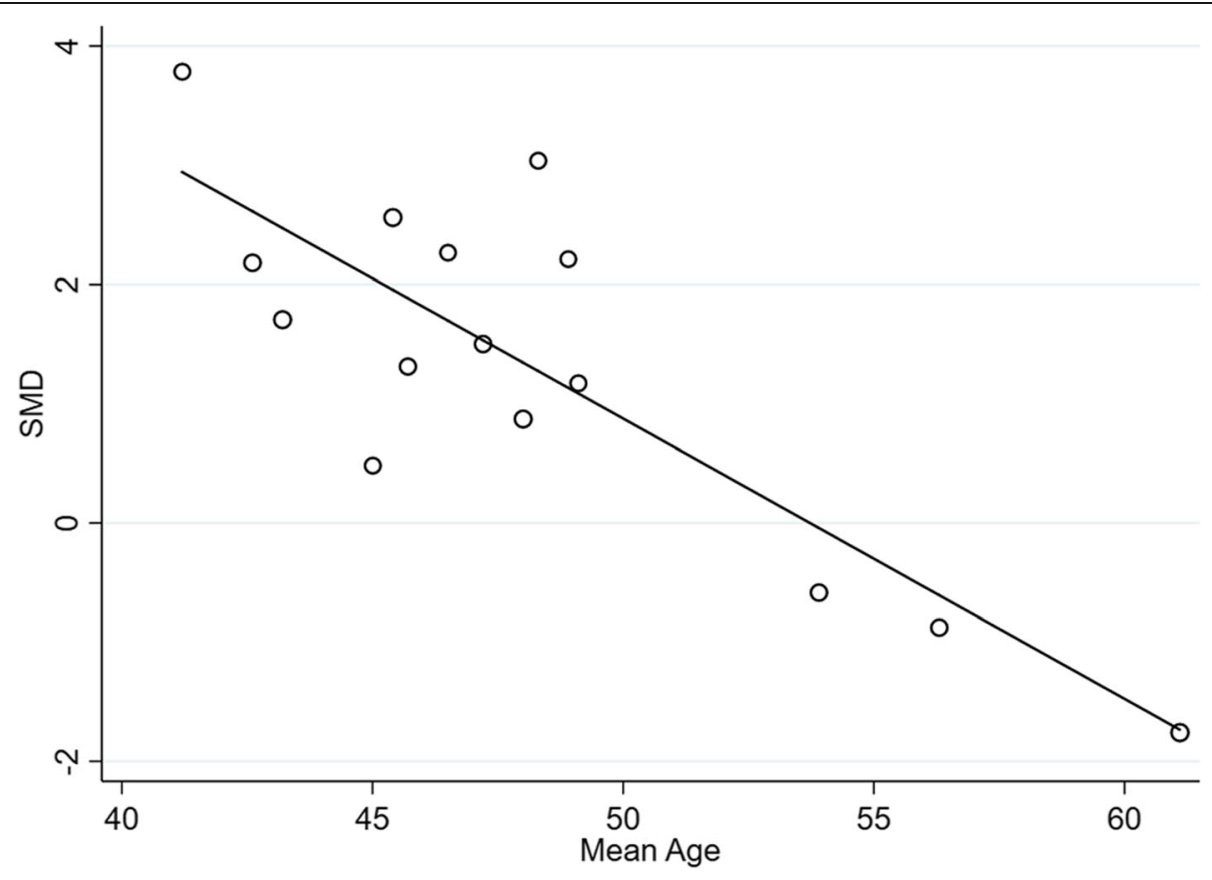

Fig. 4 Meta-regression analysis of the effect of mean age on patients with MAFLD and healthy controls 


\section{Meta-analysis estimates, given named study is omitted | Lower Cl Limit OEstimate | Upper Cl Limit}

Kukla et al. (2010)

Yilmaz et al. (2011)

Polyzos et al. (2014)

Ye et al. (2014)

zhuang et al. (2015)

Pohl et al. (2016)

Zwolak et al. (2016)

Bekaert et al. (2016)

Lai et al. (2017)

Zhang et al. (2017)

Hang et al. (2019)

Sahar et al. (2019)

Mohamed et al. (2021)

Gao et al. (2021)

Xing et al. (2021)

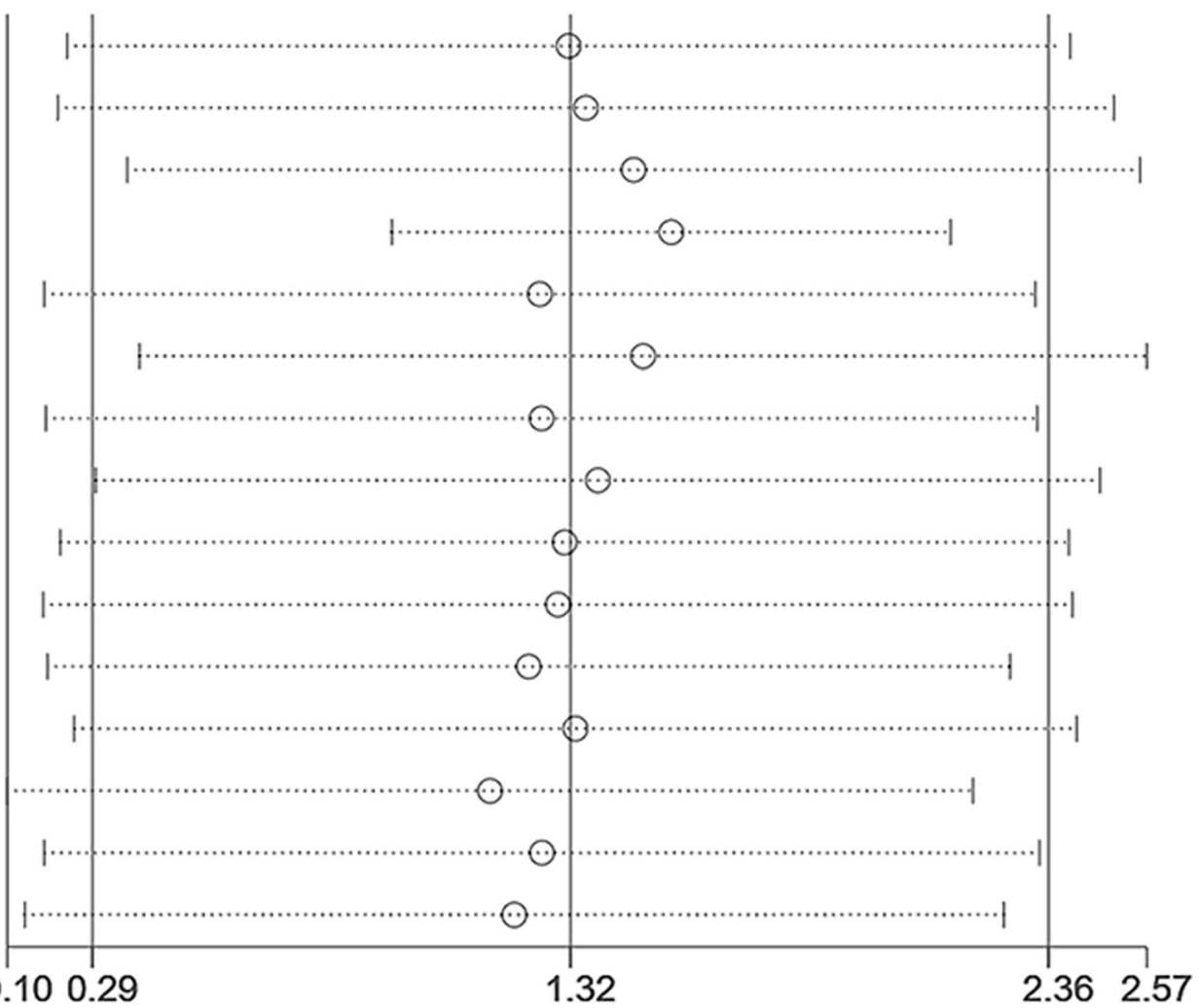

Fig. 5 Sensitivity analysis of the summary SMD on the difference in circulating chemerin levels between MAFLD patients and controls

Egger's test and funnel plots were used to determine the publication bias of the articles included in the study. The funnel plot showed significant asymmetry (Fig. S1), and Egger's test $(P=0.008)$ indicated a potential risk of publication bias. Next, using the trim and fill method, symmetric funnel plots were created, which showed that two studies were estimated to be missing. However, the results showed a significant effect after the trim-and-fill analysis (pooled effect size: 1.001; 95\% CI: 0.043, 2.049, $P=0.04)$, which confirmed the significant association of circulating chemerin with MAFLD patients in our study may not be due to publication bias.

\section{Discussion}

There is growing concern over noninvasive diagnostic indicators for MAFLD in clinical practice. Recently, circulating chemerin levels in patients with MAFLD have gained attention. A meta-analysis of chemerin levels in patients with MAFLD has been conducted for the first time. The reasons for the differences in circulating chemerin levels between MAFLD patients and healthy subjects were further summarized.
Overall, a distinct connection was found between circulating chemerin levels and MAFLD (or NAFL), although no remarkable difference was found for NASH. Higher levels of circulating chemerin were observed in patients with MAFLD (or NAFL) than in healthy controls. Indeed, chemerin levels were found to be closely linked to liver steatosis, which is in line with previous research by Levin et al. [45]. Similarly, in the human hepatoma cell line HepG2, overexpression of chemerin causes lipid accumulation [46]. In addition, one study showed that the exogenous administration of chemerin increased lipid deposition not only in the liver, but also in the kidneys and arteries [47]. This potential association may be due to the relationship between chemerin and innate immunity, which in turn is inextricably linked to MAFLD [48, 49]. Chemerin recruits immune cells to the site of tissue damage by activating its receptor CMKLR1 [49]. In addition, there is no denying that immune cell-based cytokines in the liver are essential for hepatic steatosis pathogenesis [48]. Of greater interest was a study that suggested that chemerin may be an underlying biological marker of carotid atherosclerotic plaque damage [50], and another study pointed out that 
low levels of chemerin correlate with carotid plaque stability [51]. The dual effect of chemerin may explain some of the discrepancies in this phenomenon. Chemerin acts as a proinflammatory mediator; however, it can produce a potent anti-inflammatory fragment through serine (secreted by neutrophils) or cysteine protein-cleaving enzymes (released by activated macrophages) [52]. Chemerin may be primarily associated with its anti-inflammatory effects in unstable atheromatous plaques.

A high degree of heterogeneity was identified among the studies. Because of the limitations in the number of studies, only the source of heterogeneity between MAFL D and controls was explored. However, an interesting finding was that subgroup analysis revealed that chemerin levels were higher in younger patients and lower in older patients with MAFLD. Next, the results of meta-regression showed that the mean age of MAFLD patients might be a source of heterogeneity and accounted for approximately $72.08 \%$ of the sources of heterogeneity, and circulating chemerin levels were negatively correlated with the mean age of MAFLD patients. No other sources of heterogeneity were found, but this does not rule out the possibility of other causes for heterogeneity, such as the study design and different regions. As revealed in other subgroup analyses, the case-control designs showed higher levels of chemerin; in contrast, the cross-sectional studies did not find a correlation between chemerin and MAFLD patients. The same was observed for Asian patients with MAFLD who had higher levels of chemerin. However, no correlation was observed between chemerin and MAFLD patients in other continents.

What is puzzling is that an association between chemerin levels and age has also been reported in previous studies. Coimbra et al. revealed that serum chemerin levels were increased in elderly T2DM patients [53]. Researchers found that patients with chronic heart failure who had high levels of chemerin were more likely to be older [54]. This inconsistency with our results may be closely related to a nuclear receptor-farnesoid X receptor $(F X R) . F X R$ is a key regulator of lipid and glucose metabolism that is widely distributed in the liver [55], and hepatic FXR plays a crucial role in chemerin synthesis [56]. Patients with MAFLD had lower levels of hepatic FXR expression, according to an early investigation [57]. Subsequently, Xiong et al. noted that steatosis was exacerbated by a marked reduction in $F X R$ in aging mice [58]. Therefore, we suggested that the decrease in circulating chemerin levels in elderly patients was suggested to be due to the decline of FXR receptor-mediated chemerin synthesis. Although liver steatosis was associated with high levels of circulating chemerin, it was possible that the extent of FXR downregulation in elderly patients was greater than the effect of steatosis. However, largescale studies with prospective longitudinal designs are needed to better reveal and prove this connection.

Unfortunately, no correlation was found between chemerin levels and specific liver histological lesions. However, the use of chemerin in oncology has also been emphasized. A previous report showed that chemerin expression was reduced in hepatocellular carcinoma (HCC) tissue [59]. However, Haberl et al. noted that chemerin protein levels were increased in MAFLD-associated hepatocellular tumors [60]. This paradox may be because the majority of the former study subjects had viral hepatitis as the cause of MAFLD. Equally puzzling was the finding in animal models that serum and liver chemerin were not significantly different in NASHassociated HCC compared to NASH [61]. Therefore, further clinical studies of hepatocellular carcinoma associated with MAFLD are required to determine whether circulating chemerin could be used as a predictor of hepatocellular carcinoma in MAFLD patients.

\section{Study strengths and limitation}

This study is the first systematic investigation of the association of chemerin with MAFLD, including different subtypes and specific hepatic histological lesions. A wide regional coverage of the population was involved, including Asia, Europe, and Africa. Chemerin was found to be a promising biomarker for the early detection of MAFLD, according to these findings. Several major limitations in the current study warrant consideration: First, the observational studies that were included only explored the association between chemerin and MAFLD and could not explain the causal relationship between them. Second, owing to the unavailability of large-scale prospective cohort studies, the relationship between chemerin levels and MAFLD should be interpreted with caution and considered hypothesis-generating. Third, despite attempts to explore the sources of heterogeneity, it was not possible to identify all possible heterogeneity because of the inherent differences in each participant, such as their complications and confounding factors (diabetes ratio, BMI, waist circumference, hip circumference, etc.), which potentially influenced their chemerin levels. Fourth, the mean values of circulating chemerin levels that were obtained from the different populations in this meta-analysis varied widely; therefore, SMD was used as a combined effect size. Furthermore, the MAFLD patients included in this study were not all diagnosed by liver biopsy; some were diagnosed by ultrasound. More studies of chemerin levels in patients with MAFLD who have been identified by biopsy are required in the future. 


\section{Conclusions}

To sum it up, MAFLD patients had higher levels of chemerin than healthy controls, suggesting that circulating chemokine levels may be a noninvasive potential biomarker in diagnostic applications for MAFLD patients. However, circulating chemerin levels were higher in younger MAFLD patients and lower in older patients. Large-scale studies are needed to assess circulating chemokine levels in MAFLD patients of different ages. In addition, chemerin levels did not differ statistically significantly between MAFLD subtypes or specific liver tissue lesions, and with the small sample size, results should be interpreted with caution because of the uncertainty. Future relevant studies with larger samples are needed to further explore the relationship.

\begin{abstract}
Abbreviations
ALT: alanine aminotransferase; AST: aspartate aminotransferase; BMI: body mass index; Cl: Confidence interval; HOMA-IR: homoeostasis model assessment of insulin resistance; MAFLD: metabolic-associated fatty liver disease; NAFL: nonalcoholic fatty liver; NAFLD: nonalcoholic fatty liver disease; NASH: nonalcoholic steatohepatitis; PRISMA: Preferred Reporting Items for Systematic Reviews and Meta-analysis; RARRES2: retinoic acid receptor responder 2; SD: standard deviation; SMD: standardized mean difference; T2DM: type 2 diabetes mellitus; TIG2: tazarotene inducible gene 2
\end{abstract}

\section{Supplementary information}

The online version contains supplementary material available at https://doi. org/10.1186/s12944-022-01637-7.

\section{Additional file 1 \\ Additional file 2}

\section{Acknowledgements}

Not applicable.

\section{Authors' contributions}

$\mathrm{QR}, \mathrm{HYW}, \mathrm{YX}$, and $\mathrm{WH}$ participated in the study conception and design; QR and HYW contributed to the literature search, data extraction, and quality assessment; QR, HYW, YZ and XF performed the data analysis and drafted the manuscript; and QR, YZ, XF and MW revised the manuscript. HYW, MW and ZDL performed data interpretation and revised the manuscript. $Y X$ and $\mathrm{WH}$ reviewed the manuscript and assumed primary responsibility for the final content. All authors have read and approved the final version of this manuscript.

\section{Funding}

This work was supported by grants from the Natural Science Foundation of China (Grant no. 81970676, 81800741, 82170834), and Sichuan Science and Technology Program (Grant no. 2020YFS0456).

\section{Availability of data and materials}

All data generated or analyzed during this study are included in this article. All data generated or analyzed during this study are included in this published article and its supplementary information files.

\section{Declarations}

Ethics approval and consent to participate

Not applicable.

\section{Consent for publication}

Not applicable.

\section{Competing interests}

The authors declare no conflicts of interest.

\section{Author details}

${ }^{1}$ Department of Endocrinology and Metabolism, The Affiliated Hospital of Southwest Medical University, Luzhou, Sichuan, China. ${ }^{2}$ Sichuan Clinical Research Center for Nephropathy, Luzhou, Sichuan, China. ' 2 uzhou Key Laboratory of Cardiovascular and Metabolic Diseases, the Affiliated Hospital of Southwest Medical University, Luzhou, Sichuan, China. ${ }^{4}$ Metabolic Vascular Disease Key Laboratory of Sichuan Province, Luzhou, Sichuan, China.

${ }^{5}$ Experimental Medicine Center, The Affiliated Hospital of Southwest Medical University, Luzhou, China.

Received: 3 December 2021 Accepted: 17 February 2022

Published online: 02 March 2022

\section{References}

1. Eslam M, Newsome PN, Sarin SK, Anstee QM, Targher G, Romero-Gomez M, Zelber-Sagi S, Wai-Sun Wong V, Dufour JF, Schattenberg JM, et al: A new definition for metabolic dysfunction-associated fatty liver disease: An international expert consensus statement. J Hepatol 2020, 73:202-209. doi: https://doi.org/10.1016/j.jhep.2020.03.039.

2. Clark JM, Brancati FL, Diehl AM: Nonalcoholic fatty liver disease. Gastroenterology 2002, 122:1649-1657. doi: https://doi.org/10.1053/gast.2 002.33573.

3. Younossi ZM, Koenig AB, Abdelatif D, Fazel Y, Henry L, Wymer M: Global epidemiology of nonalcoholic fatty liver disease-Meta-analytic assessment of prevalence, incidence, and outcomes. Hepatology 2016, 64:73-84. doi: https://doi.org/10.1002/hep.28431.

4. Estes C, Anstee QM, Arias-Loste MT, Bantel H, Bellentani S, Caballeria J, Colombo M, Craxi A, Crespo J, Day CP, et al: Modeling NAFLD disease burden in China, France, Germany, Italy, Japan, Spain, United Kingdom, and United States for the period 2016-2030. J Hepatol 2018, 69:896-904. doi: https://doi.org/10.1016/j.jhep.2018.05.036.

5. Friedman SL, Neuschwander-Tetri BA, Rinella M, Sanyal AJ: Mechanisms of NAFLD development and therapeutic strategies. Nat Med 2018, 24:908-922. doi: https://doi.org/10.1038/s41591-018-0104-9.

6. Byrne CD, Targher G: What's new in NAFLD pathogenesis, biomarkers and treatment? Nat Rev Gastroenterol Hepatol 2020, 17:70-71. doi: https://doi. org/10.1038/s41575-019-0239-2

7. Lonardo A, Ballestri S, Marchesini G, Angulo P, Loria P: Nonalcoholic fatty liver disease: a precursor of the metabolic syndrome. Dig Liver Dis 2015, 47: 181-190. doi: https://doi.org/10.1016/j.dld.2014.09.020.

8. Adams LA, Anstee QM, Tilg H, Targher G: Non-alcoholic fatty liver disease and its relationship with cardiovascular disease and other extrahepatic diseases. Gut 2017, 66:1138-1153. doi: https://doi.org/10.1136/gutjnl-201 7-313884.

9. Liu SS, Ma XF, Zhao J, Du SX, Zhang J, Dong MZ, Xin YN: Association between nonalcoholic fatty liver disease and extrahepatic cancers: a systematic review and meta-analysis. Lipids Health Dis 2020, 19:118. doi: https://doi.org/10.1186/s12944-020-01288-6.

10. Younossi ZM, Blissett D, Blissett R, Henry L, Stepanova M, Younossi Y, Racila A, Hunt S, Beckerman R: The economic and clinical burden of nonalcoholic fatty liver disease in the United States and Europe. Hepatology 2016, 64: 1577-1586. doi: https://doi.org/10.1002/hep.28785.

11. Rockey DC, Caldwell SH, Goodman ZD, Nelson RC, Smith AD: Liver biopsy. Hepatology 2009, 49:1017-1044. doi: https://doi.org/10.1002/hep.22742.

12. Nagpal S, Patel S, Jacobe H, DiSepio D, Ghosn C, Malhotra M, Teng M, Duvic M, Chandraratna RA: Tazarotene-induced gene 2 (TIG2), a novel retinoidresponsive gene in skin. J Invest Dermatol 1997, 109:91-95. doi: https://doi. org/10.1111/1523-1747.ep12276660.

13. Wittamer V, Franssen JD, Vulcano M, Mirjolet JF, Le Poul E, Migeotte I, Brézillon S, Tyldesley R, Blanpain C, Detheux M, et al: Specific recruitment of antigen-presenting cells by chemerin, a novel processed ligand from human inflammatory fluids. J Exp Med 2003, 198:977-985. doi: https://doi. org/10.1084/jem.20030382.

14. Ernst MC, Sinal CJ: Chemerin: at the crossroads of inflammation and obesity. Trends Endocrinol Metab 2010, 21:660-667. doi: https://doi.org/10.1016/j. tem.2010.08.001.

15. Bozaoglu K, Curran JE, Stocker CJ, Zaibi MS, Segal D, Konstantopoulos N, Morrison S, Carless M, Dyer TD, Cole SA, et al: Chemerin, a novel adipokine 
in the regulation of angiogenesis. J Clin Endocrinol Metab 2010, 95:24762485. doi: https://doi.org/10.1210/jc.2010-0042.

16. Sell H, Laurencikiene J, Taube A, Eckardt K, Cramer A, Horrighs A, Arner P, Eckel J: Chemerin is a novel adipocyte-derived factor inducing insulin resistance in primary human skeletal muscle cells. Diabetes 2009, 58:27312740. doi: https://doi.org/10.2337/db09-0277.

17. Becker M, Rabe K, Lebherz C, Zugwurst J, Göke B, Parhofer KG, Lehrke M, Broedl UC: Expression of human chemerin induces insulin resistance in the skeletal muscle but does not affect weight, lipid levels, and atherosclerosis in LDL receptor knockout mice on high-fat diet. Diabetes 2010, 59:28982903. doi: https://doi.org/10.2337/db10-0362.

18. Ernst MC, Issa M, Goralski KB, Sinal CJ: Chemerin exacerbates glucose intolerance in mouse models of obesity and diabetes. Endocrinology 2010 151:1998-2007. doi: https://doi.org/10.1210/en.2009-1098.

19. Jin $\mathrm{CH}$, Yi KW, Ha YR, Shin JH, Park HT, Kim T, Hur JY: Chemerin Expression in the Peritoneal Fluid, Serum, and Ovarian Endometrioma of Women with Endometriosis. Am J Reprod Immunol 2015, 74:379-386. doi: https://doi. org/10.1111/aji.12405.

20. Zhang O, Ji Q, Lin Y, Wang Z, Huang Y, Lu W, Liu X, Zhang J, Liu Y, Zhou YJ: Circulating chemerin levels elevated in dilated cardiomyopathy patients with overt heart failure. Clin Chim Acta 2015, 448:27-32. doi: https://doi. org/10.1016/j.cca.2015.05.018.

21. Sochal M, Fichna J, Gabryelska A, Talar-Wojnarowska R, Białasiewicz P, Małecka-Wojciesko E. Serum Levels of Chemerin in Patients with Inflammatory Bowel Disease as an Indicator of Anti-TNF Treatment Efficacy. J Clin Med. 2021;10:4615. https://doi.org/10.3390/jcm10194615.

22. Birkenfeld AL, Shulman Gl: Nonalcoholic fatty liver disease, hepatic insulin resistance, and type 2 diabetes. Hepatology 2014, 59:713-723. doi: https:// doi.org/10.1002/hep.26672

23. Gao B, Tsukamoto H. Inflammation in Alcoholic and Nonalcoholic Fatty Liver Disease: Friend or Foe? Gastroenterology. 2016;150:1704-9. https://doi. org/10.1053/j.gastro.2016.01.025.

24. Kukla M, Zwirska-Korczala K, Hartleb M, Waluga M, Chwist A, Kajor M, Ciupinska-Kajor M, Berdowska A, Wozniak-Grygiel E, Buldak R: Serum chemerin and vaspin in non-alcoholic fatty liver disease. Scand J Gastroenterol 2010, 45:235-242. doi: https://doi.org/10.3109/0036552 0903443852

25. Yilmaz Y, Yonal O, Kurt R, Alahdab YO, Eren F, Ozdogan O, Celikel CA, Imeryuz N, Kalayci C, Avsar E: Serum levels of omentin, chemerin and adipsin in patients with biopsy-proven nonalcoholic fatty liver disease. Scand J Gastroenterol 2011, 46:91-97. doi: https://doi.org/10.3109/003 65521.2010 .516452$.

26. Zhuang X, Sun F, Li L, Jiang D, Li X, Sun A, Pan Z, Lou N, Zhang L, Lou F: Therapeutic Effect of Metformin on Chemerin in Non-Obese Patients with Non-Alcoholic Fatty Liver Disease (NAFLD). Clin Lab 2015, 61:1409-1414. doi: https://doi.org/10.7754/clin.lab.2015.150211.

27. Zwolak A, Szuster-Ciesielska A, Daniluk J, Semeniuk J, Kandefer-Szerszen $\mathrm{M}$ : Chemerin, retinol binding protein- 4 , cytokeratin-18 and transgelin-2 presence in sera of patients with non-alcoholic liver fatty disease. Ann Hepatol 2016, 15:862-869. doi: https://doi.org/10.5604/16652681.12221 02.

28. Lai M, Zhang S, Jin Q, Huang J, Xu J, Zeng H, Qin Y, Wu D, Ge B, Xu G, Wu $P$ : The correlation between serum levels of IL-11 $\beta, I L-8$ and chemerinin patients with non-alcoholic fatty liver disease. Pract Clin Med 2017, 14:6466.

29. Zhang Z, Wang J, Wang H: Correlation of blood glucose, serum chemerin and insulin resistance with NAFLD in patients with type 2 diabetes mellitus. Exp Ther Med 2018, 15:2936-2940. doi: https://doi.org/10.3892/etm.2018. 5753.

30. Sahar MI, Naglaa AE, Zeinab HES, Aida AAEH, Inass HA, Azza AA. Chemerin and vaspin as noninvasive biomarkers in the pathogenesis and diagnosis of non- alcoholic fatty liver disease. Am J Med Sci. 2019:9:7.

31. Hang S, Shen H, Chen M: Correlation of serum SHGB levels with insulin sensitivity, lipid metabolism oxidative stress in patients with nonalcoholic fatty liver disese. J Clin Exp Med 2019, 18:82-85.

32. Mohamed G, Salama MM, Byoumy E, Mohamed A, Ibrahim W, El-shafie A, Abdallah M: Chemerin As A Non-Invasive Serum Marker for Non-Alcoholic Fatty Liver Disease. The Egyptian Journal of Hospital Medicine 2021, 84: 1786-1790. doi: https://doi.org/10.21608/ejhm.2021.177253.

33. Gao $H$, Yang $D$, Liang $H$, Wang $X$, Wang $Y$, Chen B: Association of serum Chemerin levels and carotid intima-media thickness in patients with nonalcoholic fatty liver disease. Chin Hepatol 2021, 26:429-434. doi: https:// doi.org/10.14000/j.cnki.issn.1008-1704.2021.04.023.

34. Xing D, Sun Y, Yu L, Su S, Zhang H: Changes of serum Chemerin level in patients with nonalcoholic fatty liver disease and its relationship with insulin resistance and inflammation. Mod Med J Chin 2021, 23:23-26. doi: https:// doi.org/10.3969/j.issn.1672-9463.2021.08.006

35. Ye Z, Wang S, Yang Z, He M, Zhang S, Zhang W, Wen J, Li Q, Huang Y, Wang $X$, et al: Serum lipocalin-2, cathepsin S and chemerin levels and nonalcoholic fatty liver disease. Mol Biol Rep 2014, 41:1317-1323. doi: https://doi.org/10.1007/s11033-013-2977-5.

36. Polyzos SA, Kountouras J, Anastasilakis AD, Geladari EV, Mantzoros CS: Irisin in patients with nonalcoholic fatty liver disease. Metabolism 2014, 63:207217. doi: https://doi.org/10.1016/j.metabol.2013.09.013.

37. Bekaert M, Ouwens DM, Hörbelt T, Van de Velde F, Fahlbusch P, Herzfeld de Wiza D, Van Nieuwenhove Y, Calders P, Praet M, Hoorens A, et al: Reduced expression of chemerin in visceral adipose tissue associates with hepatic steatosis in patients with obesity. Obesity (Silver Spring) 2016, 24:2544-2552 doi: https://doi.org/10.1002/oby.21674.

38. Pohl R, Haberl EM, Rein-Fischboeck L, Zimny S, Neumann M, Aslanidis C, Schacherer D, Krautbauer S, Eisinger K, Weiss TS, Buechler C: Hepatic chemerin mRNA expression is reduced in human nonalcoholic steatohepatitis. Eur J Clin Invest 2017, 47:7-18. doi: https://doi.org/10.1111/ eci.12695.

39. Kajor M, Kukla M, Waluga M, Liszka Ł, Dyaczyński M, Kowalski G, Żądło D, Berdowska A, Chapuła M, Kostrzab-Zdebel A, et al: Hepatic chemerin mRNA in morbidly obese patients with nonalcoholic fatty liver disease. Pol J Pathol 2017, 68:117-127. doi: https://doi.org/10.5114/pjp.2017.69687.

40. Page MJ, Moher D, Bossuyt PM, Boutron I, Hoffmann TC, Mulrow CD, Shamseer L, Tetzlaff JM, AkI EA, Brennan SE, et al: PRISMA 2020 explanation and elaboration: updated guidance and exemplars for reporting systematic reviews. Bmj 2021, 372:n160. doi: https://doi.org/10.1136/bmj.n160.

41. Morgan RL, Thayer KA, Santesso N, Holloway AC, Blain R, Eftim SE, Goldstone $A E$, Ross P, Ansari M, Akl EA, et al: A risk of bias instrument for non-randomized studies of exposures: A users' guide to its application in the context of GRADE. Environ Int 2019, 122:168-184. doi: https://doi.org/1 0.1016/j.envint.2018.11.004

42. Atkins D, Best D, Briss PA, Eccles M, Falck-Ytter Y, Flottorp S, Guyatt GH, Harbour RT, Haugh MC, Henry D, et al: Grading quality of evidence and strength of recommendations. Bmj 2004, 328:1490. doi: https://doi.org/1 0.1136/bmj.328.7454.1490.

43. Hozo SP, Djulbegovic B, Hozo I: Estimating the mean and variance from the median, range, and the size of a sample. BMC Med Res Methodol 2005, 5: 13. doi: https://doi.org/10.1186/1471-2288-5-13.

44. Sell H, Divoux A, Poitou C, Basdevant A, Bouillot JL, Bedossa P, Tordjman J, Eckel J, Clément K: Chemerin correlates with markers for fatty liver in morbidly obese patients and strongly decreases after weight loss induced by bariatric surgery. J Clin Endocrinol Metab 2010, 95:2892-2896. doi: https://doi.org/10.1210/jc.2009-2374.

45. Levin LM, Völzke H, Lerch MM, Kühn JP, Nauck M, Friedrich N, Zylla S. Associations of circulating chemerin and adiponectin concentrations with hepatic steatosis. Endocr Connect 2019, 8:1097-1107. doi: https://doi.org/1 0.1530/ec-19-0300.

46. Zhu L, Huang J, Wang Y, Yang Z, Chen X: Chemerin causes lipid metabolic imbalance and induces passive lipid accumulation in human hepatoma cell line via the receptor GPR1. Life Sci 2021, 278:119530. doi: https://doi.org/1 $0.1016 / j .1 f s .2021 .119530$.

47. Jia J, Yu F, Xiong Y, Wei W, Ma H, Nisi F, Song X, Yang L, Wang D, Yuan G, Zhou $\mathrm{H}$ : Chemerin enhances the adhesion and migration of human endothelial progenitor cells and increases lipid accumulation in mice with atherosclerosis. Lipids Health Dis 2020, 19:207. doi: https://doi.org/10.1186/ s12944-020-01378-5

48. Zhan $Y T, A n W$ : Roles of liver innate immune cells in nonalcoholic fatty liver disease. World J Gastroenterol 2010, 16:4652-4660. doi: https://doi.org/10.3 748/wjg.v16.i37.4652

49. Rourke $\mathrm{L}$, Dranse HJ, Sinal CJ: Towards an integrative approach to understanding the role of chemerin in human health and disease. Obes Rev 2013, 14:245-262. doi: https://doi.org/10.1111/obr.12009.

50. Ammirati $\mathrm{E}$, Fogacci F: Clinical relevance of biomarkers for the identification of patients with carotid atherosclerotic plaque: Potential role and limitations of cysteine protease legumain. Atherosclerosis 2017, 257:248-249. doi: https://doi.org/10.1016/j.atherosclerosis.2017.01.003. 
51. Gasbarrino K, Mantzoros C, Gorgui J, Veinot JP, Lai C, Daskalopoulou SS: Circulating Chemerin Is Associated With Carotid Plaque Instability, Whereas Resistin Is Related to Cerebrovascular Symptomatology. Arterioscler Thromb Vasc Biol 2016, 36:1670-1678. doi: https://doi.org/10.1161/atvbaha.115.3 06741.

52. Yoshimura T, Oppenheim JJ: Chemerin reveals its chimeric nature. J Exp Med 2008, 205:2187-2190. doi: https://doi.org/10.1084/jem.20081736.

53. Coimbra S, Brandão Proença J, Santos-Silva A, Neuparth MJ: Adiponectin, leptin, and chemerin in elderly patients with type 2 diabetes mellitus: a close linkage with obesity and length of the disease. Biomed Res Int 2014, 2014:701915. doi: https://doi.org/10.1155/2014/701915.

54. Zhou X, Tao Y, Chen Y, Xu W, Qian Z, Lu X: Serum Chemerin as a Novel Prognostic Indicator in Chronic Heart Failure. J Am Heart Assoc 2019, 8: e012091. doi: https://doi.org/10.1161/jaha.119.012091.

55. Sonne DP: MECHANISMS IN ENDOCRINOLOGY: FXR signalling: a novel target in metabolic diseases. Eur J Endocrinol 2021, 184:R193-r205. doi: https://doi.org/10.1530/eje-20-1410.

56. Deng Y, Wang H, Lu Y, Liu S, Zhang Q, Huang J, Zhu R, Yang J, Zhang R, Zhang $D$, et al: Identification of chemerin as a novel FXR target gene downregulated in the progression of nonalcoholic steatohepatitis. Endocrinology 2013, 154:1794-1801. doi: https://doi.org/10.1210/en.2012-2126.

57. Yang ZX, Shen W, Sun H: Effects of nuclear receptor FXR on the regulation of liver lipid metabolism in patients with non-alcoholic fatty liver disease. Hepatol Int 2010, 4:741-748. doi: https://doi.org/10.1007/s12072-010-9202-6.

58. Xiong X, Wang X, Lu Y, Wang E, Zhang Z, Yang J, Zhang H, Li X: Hepatic steatosis exacerbated by endoplasmic reticulum stress-mediated downregulation of FXR in aging mice. J Hepatol 2014, 60:847-854. doi: https://doi.org/10.1016/j.jhep.2013.12.003.

59. Lin W, Chen YL, Jiang L, Chen JK: Reduced expression of chemerin is associated with a poor prognosis and a lowed infiltration of both dendritic cells and natural killer cells in human hepatocellular carcinoma. Clin Lab 2011, 57:879-885,

60. Haberl EM, Feder S, Pohl R, Rein-Fischboeck L, Dürholz K, Eichelberger L, Wanninger J, Weiss TS, Buechler C. Chemerin Is Induced in Non-Alcoholic Fatty Liver Disease and Hepatitis B-Related Hepatocellular Carcinoma. Cancers (Basel). 2020;12:2967. https://doi.org/10.3390/cancers12102967.

61. Haberl EM, Pohl R, Rein-Fischboeck L, Feder S, Sinal CJ, Buechler C: Chemerin in a Mouse Model of Non-alcoholic Steatohepatitis and Hepatocarcinogenesis. Anticancer Res 2018, 38:2649-2657. doi: https://doi. org/10.21873/anticanres.12507.

\section{Publisher's Note}

Springer Nature remains neutral with regard to jurisdictional claims in published maps and institutional affiliations.

Ready to submit your research? Choose BMC and benefit from:

- fast, convenient online submission

- thorough peer review by experienced researchers in your field

- rapid publication on acceptance

- support for research data, including large and complex data types

- gold Open Access which fosters wider collaboration and increased citations

- maximum visibility for your research: over $100 \mathrm{M}$ website views per year

At BMC, research is always in progress.

Learn more biomedcentral.com/submissions 